\title{
Determining the Probability of Recovering Data from Damaged USB Flash Drive
}

\author{
Kalpana Shinde, Vini Kale, C.N.Kayte, Shobha Bawiskar
}

\begin{abstract}
Habit of storing digital data is becoming a common practice. To cope this need lots off secondary memory devices are commercially available in cheap prices. Important factor is significance of data, its dependability in human daily life. Hence looking at this scenario the cyber crime rates are at hike. Mostly after committing the crime intentionally or unintentionally criminals try to destroy the digital evidence by doing damage to $e$ - device. Basic aim is to check whether damaged secondary can recover the data or not. For this purpose various damaged setup are done and by using software's results are analyzed.
\end{abstract}

Keywords: Pen Drive(PD), Pen Drive Models, Data Recovery, Damaged PD

\section{INTRODUCTION}

Secondary storage media are widely used as it is portable, small/micro in size, light weighted and easily carried from one place to another, Consumes less power compared to other devices.[21] Universal USB, less expensive. . The USB which stands for Universal Serial Bus is already a

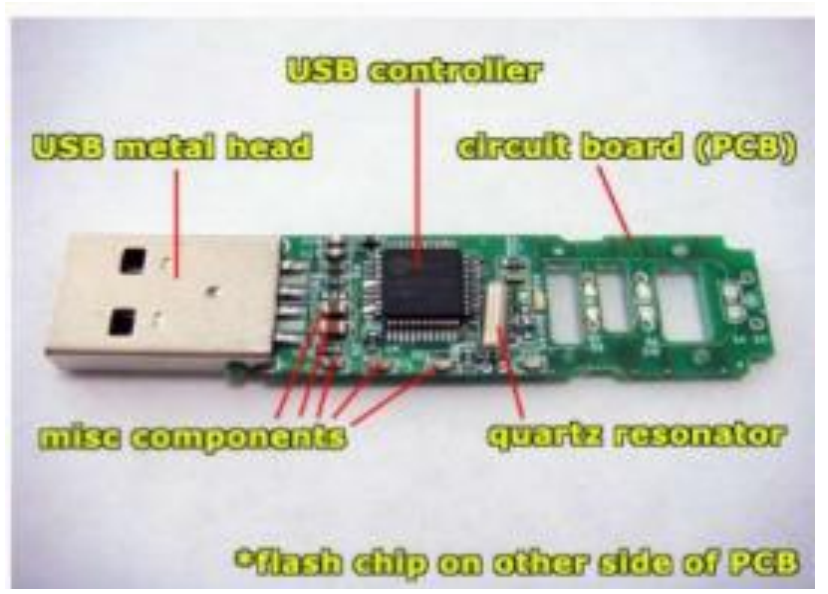

Revised Version Manuscript Received on Jun 20, 2019.

Kalpana Dnyaneshwar Shinde, Student of M.Sc (Forensic Science), Government Institute of Forensics Science Aurangabad, Department of Digital and Cyber Forensics.

Kale Vini Arun, Assistant Professor in Government Institute of Forensic Science. Aurangabad.

Charansing Nathusing Kayte, HOD of Digital and Cyber forensic in Government Institute of Forensic Science. Aurangabad.

Shobha Bawiskar, Assistance Professor in Government Institute of Forensic Science. Aurangabad. customary in business for attaching peripheral devices to different types of computer $[17,18]$. In this article A pen drive, or a USB flash drive is used for digital investigation. In market currently available pen drives with storage capacities ranging from $4 \mathrm{~GB}$ and $32 \mathrm{~GB}, 64 \mathrm{~GB}, \ldots$ can be used to store multimedia content like graphics-heavy documents, photos, music files and video clips of various file formats. [19, 20,]As of March 2016, flash drives with anywhere from 8 to $256 \mathrm{~GB}$ were frequently sold, while $512 \mathrm{~GB}$ and $1 \mathrm{~TB}$ units were less frequent [13,14].As of 2018, 2TB flash drives were the largest available in terms of storage capacity.[15]. The extensive market for USB flash drives which is projected to exceed 555 million units by the year 2020 had shown the increasing importance of the technology in consumer applications [16]

\section{PEN DRIVE}

Design and implementation1. USB standard male-A plug 2.USB mass storage control device. 3. Test points. 4. Flash memory chip. 5. Crystal oscillator 6.LED (optional). 7. Write protect switch (option). 8. Space for second flash memory[22,23]

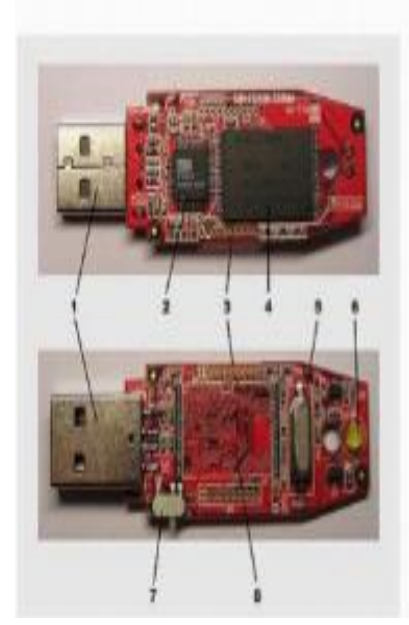

1.USB standard male-A plug
2.USB mass storage control
device.
3.Test points.
4.Flash memory chip.
5.Crystal oscillator
6.LED(optional).
7.Write protect switch(option),
8.Space for second flash
memory.

\section{PEN DRIVE MODELS:}

Now a day's storage devices, recording devices, spy camera are present in disguised manner. It's just unbelievable that such devices exits can be used for or against any persons either to save or destroy human property.(may be tangible or intangible). Cyber Crime is technology based crime committed by technocrats.[12] 
As this research article is fo0cused on pen drives few disguised pen drive models are shown below.

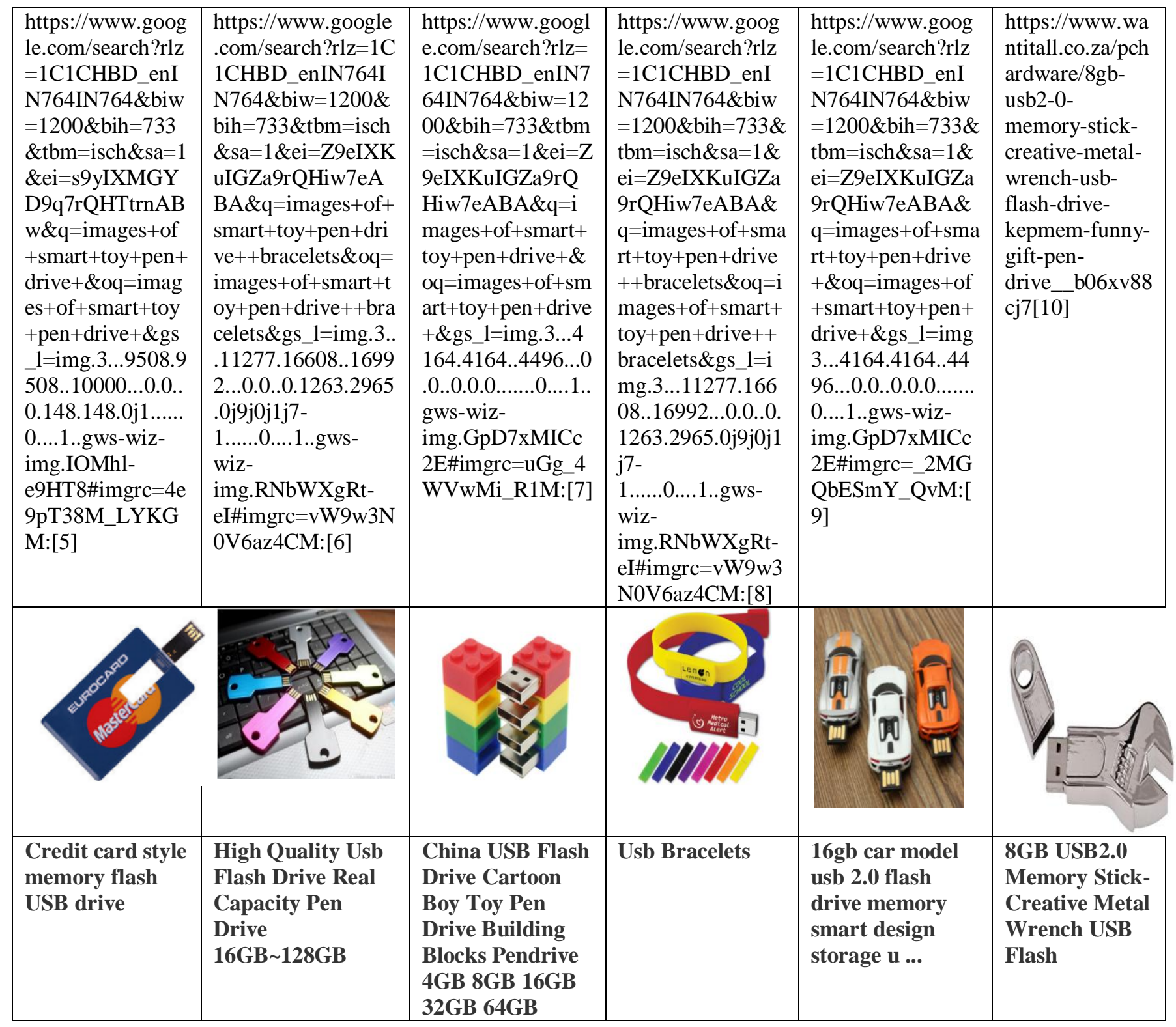




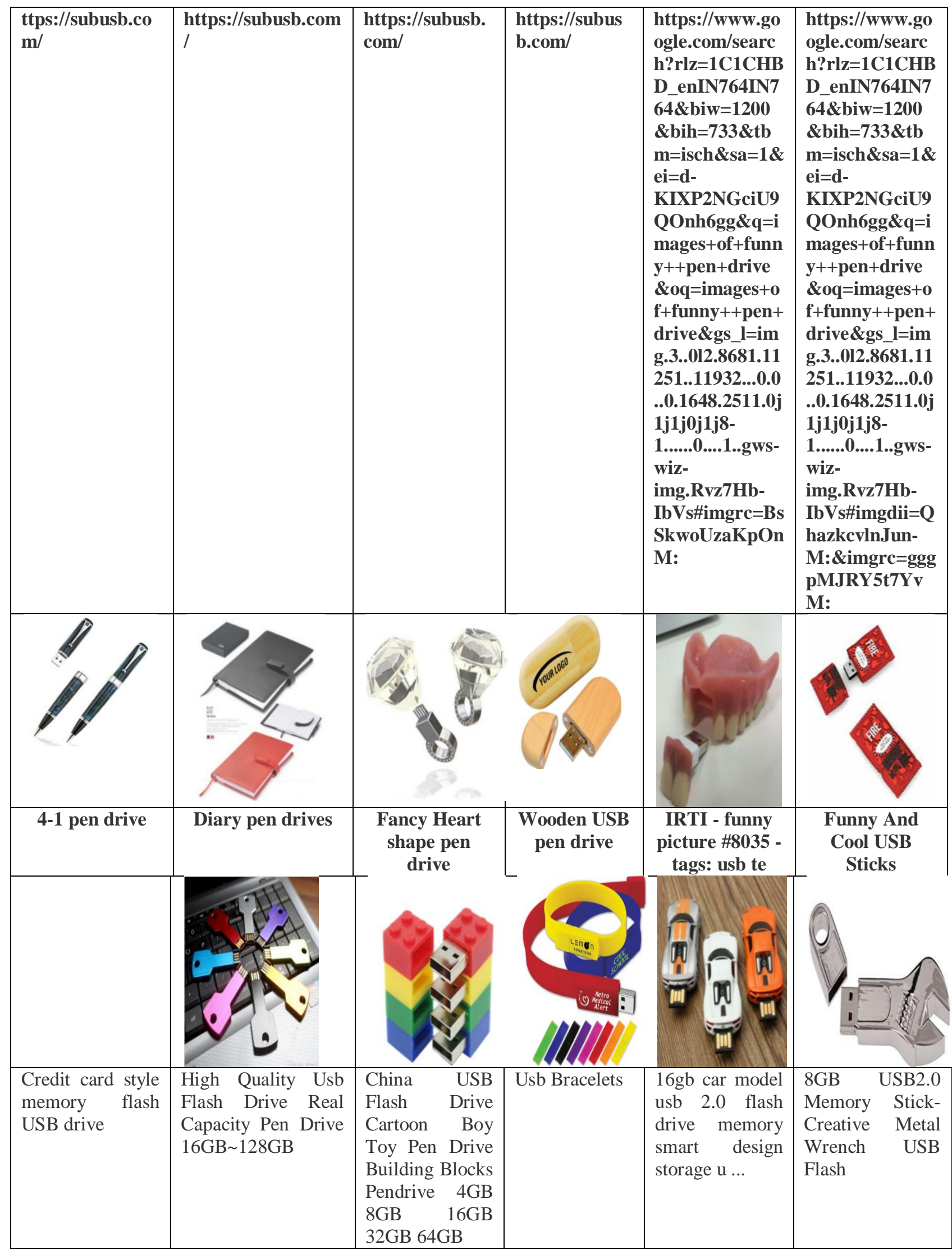




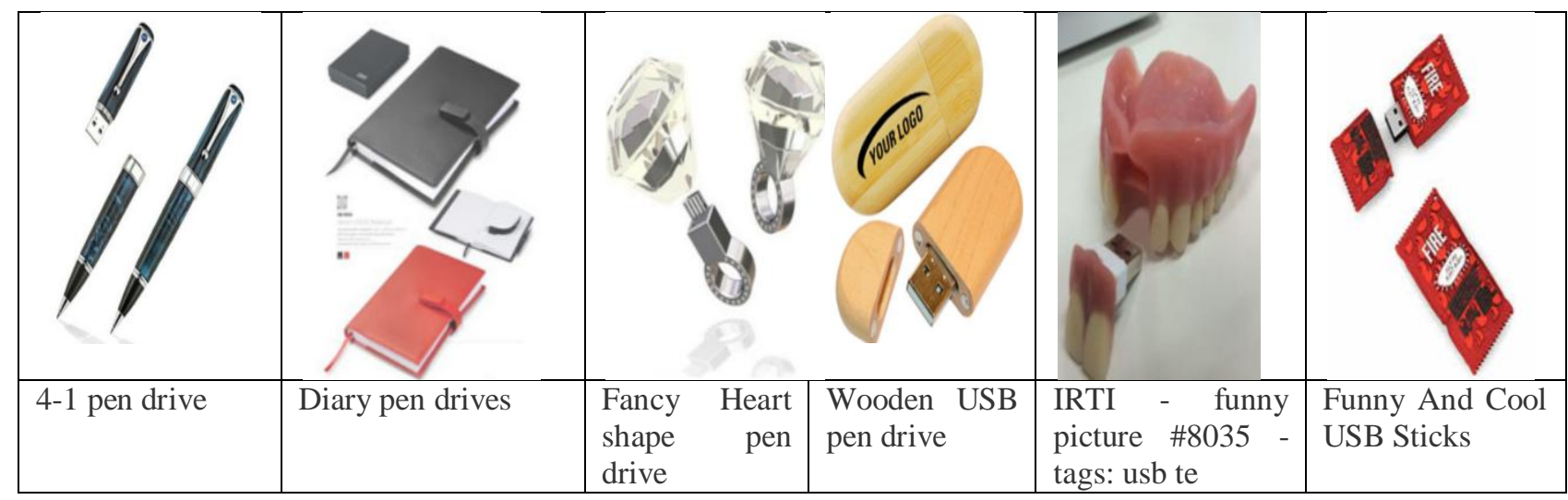

Data loss, data deletion, device formatting or physical damage to PD may be accidentally or unintentionally happen. In this scenario role of data is important. Hence data recovery can be possible in above mentioned criteria's. this article majorly deals with physically damaging PD and then check for data recovery possibilities.

\section{A. Data Recovery}

The information remaining on the storage can be recovered to a safe location. Recovery chances depend greatly on the specific data loss situation Data recovery software serves to get data back after its loss with the maximum result possible. Commonly, data recovery process is based on storage scan to find specific information (deleted files, lost file systems) and assemble structures of the damaged file system.

The process of retrieving and handling the data from damaged, failed, corrupted or inaccessible secondary storage device when it is unable to access it normally is known as file recovery process.[11]

\section{B. Recovery data process is done in three steps:}

1. Recovery Software first detects the device PD

2. Scan the device (select either 2.1 or 2.2)

3. for this article 2.2 is selected2.1 Quick Scan Searches for deleted files and folders using a basic algorithm for fast results.

2.2 Deep Scan - Scans the storage device sector by sector to ensure recovery results.

4. Recover the data can be $\mathrm{F}=$ Fully recovered ,P=partially Recovered, NO=No Recovery..3.1Preview all files available for recovery.3.2 Choose what you want to recover to avoid unnecessary recovery.

\section{EXPERIMENT:-}

A. Data Sample: Multimedia content of one GB (1GB data) with Maximum types of file extensions are collected as data sample.

$B$. Device used: 25 SanDisk's Cruzer Blade ${ }^{\mathrm{TM}}$ USB Flash Drive of 16 GB (PD).

2.1 Method 1: Buried in Mud Method

\begin{tabular}{|l|l|l|l|l|}
\hline Sample No & Mud & Time Factor & Detectable & $\begin{array}{l}\text { Recovery } \\
\text { possibility }\end{array}$ \\
\hline Sample 1 & Dry & $1 \mathrm{hr}$ & yes & yes \\
\hline Sample 2 & Dry & 1-day= 24hrs & yes & yes \\
\hline Sample 3 & Semi wet & $1 \mathrm{hr}$ & yes & yes \\
\hline Sample 4 & Semi Wet & $5 \mathrm{hrs}$ & yes & yes \\
\hline Sample 5 & Liquid muddy & 7 -days=168 hrs & yes & yes \\
\hline
\end{tabular}


2.2 Method 2: Use of aqueous media like Water,

\begin{tabular}{|c|c|c|c|c|c|c|}
\hline $\begin{array}{l}\text { Sample } \\
\text { No }\end{array}$ & $\begin{array}{l}\text { Water } \\
\text { amount }\end{array}$ & $\begin{array}{l}\text { Water } \\
\text { Type }\end{array}$ & $\begin{array}{l}\text { Environment } \\
\text { temperature } \\
\text { min } \\
\text { approximately }\end{array}$ & $\begin{array}{l}\text { Time } \\
\text { depth } \\
\text { Factor }\end{array}$ & $\begin{array}{l}\text { Detecte } \\
\text { d }\end{array}$ & $\begin{array}{l}\text { Recovery } \\
\text { possibility }\end{array}$ \\
\hline $\begin{array}{l}\text { Sample } \\
1\end{array}$ & $\begin{array}{l}4 \text { Itr in } \\
\text { bucket }\end{array}$ & Normal Water & 6-8 & $1 \mathrm{hr}$ & yes & yes \\
\hline $\begin{array}{l}\text { Sample } \\
2\end{array}$ & $\begin{array}{l}4 \quad \text { Itr in } \\
\text { bucket }\end{array}$ & Normal Water & $8-11$ & 12 hrs & yes & yes \\
\hline $\begin{array}{l}\text { Sample } \\
3\end{array}$ & $\begin{array}{l}500 \\
\text { bottle }\end{array}$ & Drainage Water & 8-14 & $12 \mathrm{hrs}$ & yes & yes \\
\hline $\begin{array}{l}\text { Sample } \\
4\end{array}$ & $\begin{array}{l}500 \mathrm{ml} \text { in } \\
\text { beaker }\end{array}$ & $\begin{array}{l}\text { Hot } 50 \text { continuously } \\
\text { boiled Water }\end{array}$ & 11-14 & $1 \mathrm{hr}$ & yes & yes \\
\hline $\begin{array}{l}\text { Sample } \\
5\end{array}$ & $\begin{array}{l}500 \text { ml in } \\
\text { beaker }\end{array}$ & $\begin{array}{l}\text { Hot } 100 \text { continuously } \\
\text { boiled Water }\end{array}$ & 11-14 & $1 \mathrm{hr}$ & No & No \\
\hline
\end{tabular}

2.3 Method 3: Heating Method

\begin{tabular}{|l|l|l|l|l|l|}
\hline Sample No & $\begin{array}{l}\text { Heating equipment } \\
\text { used }\end{array}$ & $\begin{array}{l}\text { Temperature } \\
\text { in Celsius }\end{array}$ & Time Factor & Detectable & $\begin{array}{l}\text { Recovery } \\
\text { possibility }\end{array}$ \\
\hline Sample 1 & Hot Oven Method & $\mathbf{5 0}$ & $1 \mathrm{hr}$ & yes & yes \\
\hline Sample 2 & Hot Oven Method & $\mathbf{8 0}$ & $1 \mathrm{hr}$ & yes & yes \\
\hline Sample 3 & Hot Oven Method & $\mathbf{1 6 0}$ & $1 \mathrm{hr}$ & yes & yes \\
\hline Sample 4 & Bunsen Burner & heated & $\mathbf{0 5} \mathrm{sec}$ & yes & yes \\
\hline Sample 5 & Bunsen Burner & heated & $60 \mathrm{~min}$ & Yes/no & no \\
\hline
\end{tabular}

\subsection{Method 4: Freezing Method}

\begin{tabular}{|l|l|l|l|l|}
\hline Sample No & $\begin{array}{l}\text { Freezer } \\
\text { Temperature in } \\
\text { degree Celsius }\end{array}$ & $\begin{array}{l}\text { Time } \\
\text { Factor }\end{array}$ & Detectable & Recovery possibility \\
\hline Sample 1 & -20 & 1 & yes & yes \\
\hline Sample 2 & -20 & 24 & yes & yes \\
\hline Sample 3 & -20 & 48 & yes & yes \\
\hline Sample 4 & -20 & 72 & yes & yes \\
\hline Sample 5 & -20 & 96 & yes & yes \\
\hline
\end{tabular}


Determining the Probability of Recovering Data from Damaged USB Flash Drive

2.5 Method 5: Scratching Method

\begin{tabular}{|l|l|l|l|}
\hline Sample No & No of Scratches with I-pin & Detectable & Recovery possibility \\
\hline Sample 1 & 100 & yes & yes \\
\hline Sample 2 & 150 & yes & yes \\
\hline Sample 3 & 200 & yes & yes \\
\hline Sample 4 & 250 & yes & yes \\
\hline Sample 5 & 500 & no & no \\
\hline
\end{tabular}

\section{Observation}

Method: Water Deep Method

\begin{tabular}{|c|c|c|c|c|c|c|c|c|c|c|}
\hline \multirow{6}{*}{$\begin{array}{l}\text { Recuv } \\
\mathbf{a}[1]\end{array}$} & $\begin{array}{l}\text { No of } \\
\text { samples }\end{array}$ & $\begin{array}{l}\text { Type } \\
\text { s of } \\
\text { files } \\
\text { in }\end{array}$ & $\begin{array}{l}\text { No. } \\
\text { files } \\
\text { before } \\
\text { recover }\end{array}$ & $\begin{array}{l}\text { Scanning } \\
\text { status }\end{array}$ & $\begin{array}{l}\text { No files } \\
\text { after } \\
\text { recover }\end{array}$ & $\begin{array}{l}\text { Noise } \\
\text { And type of noise } \\
\& \\
\text { Reason of noise }\end{array}$ & $\begin{array}{l}\text { Data } \\
\text { loss }\end{array}$ & $\begin{array}{l}\text { Time } \\
\text { required for } \\
\text { deep } \\
\text { scanning }\end{array}$ & $\begin{array}{l}\text { Type of } \\
\text { recovery } \\
\text { F/P/NO } \\
\text { recovery }\end{array}$ & $\begin{array}{l}\text { Recovered } \\
\text { Data }\end{array}$ \\
\hline & $\begin{array}{l}1 \mathrm{HR} \text { in } \\
\text { clean } \\
\text { water }\end{array}$ & \multirow{5}{*}{$\begin{array}{l}\text { Webp } \\
\text {,dll,ex } \\
\text { e,m4a } \\
\text {,mp3, } \\
\text { jpg,p } \\
\text { ng,pp } \\
\text { tx,doc } \\
\text { s,pdf, } \\
\text { mkv, } \\
\text { mp4 }\end{array}$} & 269 & Successful & 300 & \multirow{4}{*}{$\begin{array}{l}\text { Types of noise: } \\
\text { Wed,To,TED,OY, } \\
\text { OUT,CESa,ITH,IO } \\
\text { N,ING,ILE,IA,I<, } \\
\text { HFA,H<(E,H<,Fix, } \\
\text { File,EAD,ATE,AT } \\
\text { A,AND,AFL,\%f",s } \\
\text { ",SD }\end{array}$} & $\mathrm{N}$ & $2.40 \mathrm{HR}$ & $\mathrm{F}$ & $33 \mathrm{~GB}$ \\
\hline & $\begin{array}{l}12 \mathrm{HR} \\
\text { in clean } \\
\text { water }\end{array}$ & & 269 & Successful & 300 & & $\mathrm{~N}$ & $1.25 \mathrm{HR}$ & $\mathrm{F}$ & $33 \mathrm{~GB}$ \\
\hline & $\begin{array}{l}12 \mathrm{HR} \\
\text { dirty } \\
\text { water }\end{array}$ & & 269 & Successful & 300 & & $\mathrm{~N}$ & $2.10 \mathrm{HR}$ & $\mathrm{F}$ & $33 \mathrm{~GB}$ \\
\hline & $\begin{array}{l}1.15 \mathrm{HR} \\
\text { in water } \\
\text { at } 60 \\
\text { degree }\end{array}$ & & 269 & Successful & 299 & & $\mathrm{~N}$ & $1.5 \mathrm{HR}$ & $\mathrm{F}$ & $33 \mathrm{~GB}$ \\
\hline & $\begin{array}{l}1 \mathrm{HR} \\
\text { boiling } \\
\text { water }\end{array}$ & & 269 & \multicolumn{5}{|c|}{ NOT DETECTED } & $\mathrm{N}$ & 00 \\
\hline & & & & & & & & & & \\
\hline \multirow{5}{*}{$\begin{array}{l}\text { 7datar } \\
\text { ec[2] }\end{array}$} & $\begin{array}{l}1 \mathrm{HR} \text { in } \\
\text { clean } \\
\text { water }\end{array}$ & \multirow{5}{*}{$\begin{array}{l}\text { Webp } \\
\text {,dll,ex } \\
\text { e,m4a } \\
\text {,mp3, } \\
\text { jpg,p } \\
\text { ng,pp } \\
\text { tx,doc } \\
\text { s,pdf, } \\
\text { mkv, } \\
\text { mp4 }\end{array}$} & 269 & Successful & 505 & \multirow{4}{*}{$\begin{array}{l}\text { Repeated data get } \\
\text { recovered. } \\
\text { Noise:\#.OUT file }\end{array}$} & No & $2.15 \mathrm{HR}$ & $\mathrm{F}$ & $1.70 \mathrm{~GB}$ \\
\hline & $\begin{array}{l}12 \mathrm{HR} \\
\text { in clean } \\
\text { water }\end{array}$ & & 269 & Successful & 505 & & No & $1.56 \mathrm{HR}$ & $\mathrm{F}$ & $1.70 \mathrm{~GB}$ \\
\hline & $\begin{array}{l}12 \mathrm{HR} \\
\text { dirty } \\
\text { water }\end{array}$ & & 269 & Successful & 505 & & No & $2.16 \mathrm{HR}$ & $\mathrm{F}$ & $1.70 \mathrm{~GB}$ \\
\hline & $\begin{array}{l}1.15 \mathrm{HR} \\
\text { in water } \\
\text { at } 60 \\
\text { degree }\end{array}$ & & 269 & Successful & 500 & & No & $2.45 \mathrm{HR}$ & $\mathrm{F}$ & $1.70 \mathrm{~GB}$ \\
\hline & $\begin{array}{l}1 \mathrm{HR} \\
\text { boiling } \\
\text { water }\end{array}$ & & 269 & \multicolumn{5}{|c|}{ NOT DETECTED } & $\mathrm{N}$ & 00 \\
\hline & & & & & & & & & & \\
\hline Photor & $\begin{array}{l}1 \mathrm{HR} \text { in } \\
\text { clean } \\
\text { water }\end{array}$ & & 269 & Successful & 274 & & No & $1.67 \mathrm{HRS}$ & $\mathrm{F}$ & $997 \mathrm{MB}$ \\
\hline
\end{tabular}




\begin{tabular}{|c|c|c|c|c|c|c|c|c|c|c|}
\hline \multirow[t]{4}{*}{ ec[3] } & $\begin{array}{l}12 \mathrm{HR} \\
\text { in clean } \\
\text { water }\end{array}$ & \multirow{4}{*}{$\begin{array}{l}\text { Webp } \\
\text {,dll,ex } \\
\text { e,m4a } \\
\text {,mp3, } \\
\text { jpg,p } \\
\text { ng,pp } \\
\text { tx,doc } \\
\text { s,pdf, } \\
\text { mkv, } \\
\text { mp4 }\end{array}$} & 269 & Successful & 274 & \multirow[t]{3}{*}{$\begin{array}{l}\text { NO noise } \\
\text { observed. }\end{array}$} & No & $1.30 \mathrm{HRS}$ & $\mathrm{F}$ & $997 \mathrm{MB}$ \\
\hline & $\begin{array}{l}12 \mathrm{HR} \\
\text { dirty } \\
\text { water }\end{array}$ & & 269 & Successful & 274 & & No & $1.55 \mathrm{HRS}$ & $\mathrm{F}$ & $997 \mathrm{MB}$ \\
\hline & $\begin{array}{l}1.15 \mathrm{HR} \\
\text { in water } \\
\text { at } 60 \\
\text { degree }\end{array}$ & & 269 & Successful & 274 & & No & $1.57 \mathrm{HRS}$ & $\mathrm{F}$ & 997MB \\
\hline & $\begin{array}{l}1 \mathrm{HR} \\
\text { boiling } \\
\text { water }\end{array}$ & & 269 & \multicolumn{5}{|c|}{ NOT DETECTED } & $\mathrm{N}$ & 00 \\
\hline & & & & & & & & & & \\
\hline \multirow{5}{*}{$\begin{array}{l}\text { Stellar } \\
{[4]}\end{array}$} & $\begin{array}{l}1 \mathrm{HR} \text { in } \\
\text { clean } \\
\text { water }\end{array}$ & \multirow{5}{*}{$\begin{array}{l}\text { Webp } \\
\text {,dll,ex } \\
\text { e,m4a } \\
\text {,mp3, } \\
\text { jpg,p } \\
\text { ng,pp } \\
\text { tx,doc } \\
\text { s,pdf, } \\
\text { mkv, } \\
\text { mp4 }\end{array}$} & 269 & Successful & 270 & \multirow[t]{4}{*}{----------- } & No & $2.58 \mathrm{hrs}$ & $\mathrm{F}$ & $1 \mathrm{~GB}$ \\
\hline & $\begin{array}{l}12 \mathrm{HR} \\
\text { in clean } \\
\text { water }\end{array}$ & & 269 & Successful & 270 & & No & $1.45 \mathrm{hrs}$ & $\mathrm{F}$ & $1 \mathrm{~GB}$ \\
\hline & $\begin{array}{l}12 \mathrm{HR} \\
\text { dirty } \\
\text { water } \\
\end{array}$ & & 269 & Successful & 270 & & No & $3.15 \mathrm{hrs}$ & $\mathrm{F}$ & $1 \mathrm{~GB}$ \\
\hline & $\begin{array}{l}1.15 \mathrm{HR} \\
\text { in water } \\
\text { at } 60 \\
\text { degree }\end{array}$ & & 269 & Successful & 270 & & No & $2.45 \mathrm{hrs}$ & $\mathrm{F}$ & $1 \mathrm{~GB}$ \\
\hline & $\begin{array}{l}1 \mathrm{HR} \\
\text { boiling } \\
\text { water }\end{array}$ & & 269 & \multicolumn{5}{|c|}{ NOT DETECTED } & $\mathrm{N}$ & 00 \\
\hline
\end{tabular}

Method: Buried Method

\begin{tabular}{|c|c|c|c|c|c|c|c|c|c|c|}
\hline \multirow{6}{*}{ recuva } & $\begin{array}{l}\text { No of } \\
\text { samples }\end{array}$ & $\begin{array}{l}\text { Types } \\
\text { of } \\
\text { files } \\
\text { in }\end{array}$ & $\begin{array}{l}\text { No files } \\
\text { before } \\
\text { recover }\end{array}$ & $\begin{array}{l}\text { Scannin } \\
\text { g status }\end{array}$ & $\begin{array}{l}\text { No files } \\
\text { after } \\
\text { recover }\end{array}$ & $\begin{array}{l}\text { Noise } \\
\text { And type of } \\
\text { noise\& } \\
\text { Reason of noise }\end{array}$ & $\begin{array}{l}\text { Data } \\
\text { loss }\end{array}$ & $\begin{array}{l}\text { Time } \\
\text { required } \\
\text { for deep } \\
\text { scanning }\end{array}$ & $\begin{array}{l}\text { Type of } \\
\text { recovery } \\
\text { F/P/NO } \\
\text { recovery }\end{array}$ & $\begin{array}{l}\text { Recovered } \\
\text { Data }\end{array}$ \\
\hline & $\begin{array}{l}1 \mathrm{HR} \\
\text { Land }\end{array}$ & \multirow{5}{*}{$\begin{array}{l}\text { Webp, } \\
\text { dll,exe } \\
\text {,m4a, } \\
\text { mp3,j } \\
\text { pg,png } \\
\text {,pptx,d } \\
\text { ocs,pd } \\
\text { f,mkv, } \\
\text { mp4 }\end{array}$} & 269 & $\begin{array}{l}\text { Successf } \\
\text { ul }\end{array}$ & 303 & \multirow{5}{*}{$\begin{array}{l}\text { Types of noise: } \\
\text { Wed,To,TED,OY } \\
\text {,OUT,CESa,ITH,I } \\
\text { ON,ING,ILE,IA,I } \\
\text { <,HFA,H<(E,H<, } \\
\text { Fix,File,EAD,AT } \\
\text { E,ATA,AND,AF } \\
\text { L,\%f",,s",SD }\end{array}$} & No & $2.54 \mathrm{hr}$ & $\mathrm{F}$ & $33 \mathrm{~GB}$ \\
\hline & 24 Land & & 269 & $\begin{array}{l}\text { Successf } \\
\text { ul }\end{array}$ & 300 & & No & $1.45 \mathrm{hrs}$ & $\mathrm{F}$ & $33 \mathrm{~GB}$ \\
\hline & $\begin{array}{l}1 \mathrm{HR} \\
\text { Mud }\end{array}$ & & 269 & $\begin{array}{l}\text { Successf } \\
\text { ul }\end{array}$ & 300 & & No & $1.43 \mathrm{hrs}$ & $\mathrm{F}$ & $33 \mathrm{~GB}$ \\
\hline & $\begin{array}{l}5 \mathrm{HR} \\
\text { mud }\end{array}$ & & 269 & $\begin{array}{l}\text { Successf } \\
\text { ul }\end{array}$ & 300 & & No & $1.43 \mathrm{hrs}$ & F & $33 \mathrm{~GB}$ \\
\hline & $\begin{array}{l}25 \mathrm{HR} \\
\text { mud }\end{array}$ & & 269 & $\begin{array}{l}\text { Successf } \\
\text { ul }\end{array}$ & 287 & & No & $1.45 \mathrm{hrs}$ & $\mathrm{F}$ & $29.5 \mathrm{~GB}$ \\
\hline \multirow{3}{*}{ 7datarec } & $\begin{array}{l}1 \mathrm{HR} \\
\text { Land }\end{array}$ & \multirow{3}{*}{$\begin{array}{l}\text { Webp, } \\
\text { dll,exe }\end{array}$} & 269 & $\begin{array}{l}\text { Successf } \\
\text { ul }\end{array}$ & 505 & \multirow{3}{*}{$\begin{array}{l}\text { Repeated data get } \\
\text { recovered. } \\
\text { Noise:\#.OUT file }\end{array}$} & No & $2.10 \mathrm{hrs}$ & $\mathrm{F}$ & $1.70 \mathrm{~GB}$ \\
\hline & 24 Land & & 269 & $\begin{array}{l}\text { Successf } \\
\text { ul }\end{array}$ & 505 & & No & $1,56 \mathrm{hrs}$ & $\mathrm{F}$ & $1.70 \mathrm{~GB}$ \\
\hline & $\begin{array}{l}1 \mathrm{HR} \\
\text { Mud }\end{array}$ & & 269 & $\begin{array}{l}\text { Successf } \\
\text { ul }\end{array}$ & 500 & & No & $2.19 \mathrm{hrs}$ & $\mathrm{F}$ & $1.70 \mathrm{~GB}$ \\
\hline
\end{tabular}


Determining the Probability of Recovering Data from Damaged USB Flash Drive

\begin{tabular}{|c|c|c|c|c|c|c|c|c|c|c|}
\hline & $\begin{array}{l}5 \mathrm{HR} \\
\text { mud }\end{array}$ & \multirow{2}{*}{$\begin{array}{l}\text { mp3,j } \\
\text { pg,png } \\
\text {,pptx,d } \\
\text { ocs,pd } \\
\text { f,mkv, } \\
\text { mp4 }\end{array}$} & 269 & $\begin{array}{l}\text { Successf } \\
\mathrm{ul}\end{array}$ & 500 & & No & 2.hrs & $\mathrm{F}$ & $1.70 \mathrm{~GB}$ \\
\hline & $\begin{array}{l}25 \mathrm{HR} \\
\text { mud }\end{array}$ & & 269 & $\begin{array}{l}\text { Successf } \\
\text { ul }\end{array}$ & 500 & & No & $2.40 \mathrm{hrs}$ & $\mathrm{F}$ & $1.70 \mathrm{~GB}$ \\
\hline \multirow{6}{*}{ photorec } & $\begin{array}{l}1 \mathrm{HR} \\
\text { Land }\end{array}$ & \multirow{5}{*}{$\begin{array}{l}\text { Webp, } \\
\text { dll,exe } \\
\text {,m4a, } \\
\text { mp3,j } \\
\text { pg,png } \\
\text {,pptx,d } \\
\text { ocs,pd } \\
\text { f,mkv, } \\
\text { mp4 }\end{array}$} & 269 & $\begin{array}{l}\text { Successf } \\
\mathrm{ul}\end{array}$ & 274 & \multirow{5}{*}{ 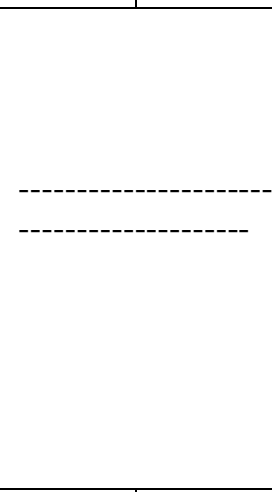 } & No & $1.56 \mathrm{hrs}$ & $\mathrm{F}$ & 997MB \\
\hline & 24 Land & & 269 & $\begin{array}{l}\text { Successf } \\
\mathrm{ul}\end{array}$ & 274 & & No & $1.50 \mathrm{hrs}$ & $\mathrm{F}$ & 997MB \\
\hline & $\begin{array}{l}1 \mathrm{HR} \\
\text { Mud }\end{array}$ & & 269 & $\begin{array}{l}\text { Successf } \\
\mathrm{ul}\end{array}$ & 274 & & No & $1.57 \mathrm{hrs}$ & $\mathrm{F}$ & $997 \mathrm{MB}$ \\
\hline & $\begin{array}{l}5 \mathrm{HR} \\
\text { mud }\end{array}$ & & 269 & $\begin{array}{l}\text { Successf } \\
\text { ul }\end{array}$ & 274 & & No & $2.14 \mathrm{hrs}$ & $\mathrm{F}$ & $997 \mathrm{MB}$ \\
\hline & $\begin{array}{l}25 \mathrm{HR} \\
\text { mud }\end{array}$ & & 269 & $\begin{array}{l}\text { Successf } \\
\text { ul }\end{array}$ & 274 & & No & $1.45 \mathrm{hrs}$ & $\mathrm{F}$ & $997 \mathrm{MB}$ \\
\hline & & & & & & & & & & \\
\hline \multirow{5}{*}{ Stellar } & $\begin{array}{l}1 \mathrm{HR} \\
\text { Land }\end{array}$ & \multirow{5}{*}{$\begin{array}{l}\text { Webp, } \\
\text { dll,exe } \\
\text {,m4a, } \\
\text { mp3,j } \\
\text { pg,png } \\
\text {,pptx,d } \\
\text { ocs,pd } \\
\text { f,mkv, } \\
\text { mp4 }\end{array}$} & 269 & $\begin{array}{l}\text { Successf } \\
\mathrm{ul}\end{array}$ & 270 & \multirow[t]{5}{*}{\begin{tabular}{|l}
- \\
-
\end{tabular}} & No & $2.16 \mathrm{hrs}$ & $\mathrm{F}$ & $1.0 \mathrm{~GB}$ \\
\hline & 24 Land & & 269 & $\begin{array}{l}\text { Successf } \\
\text { ul }\end{array}$ & 270 & & No & $2.15 \mathrm{hrs}$ & $\mathrm{F}$ & $1.0 \mathrm{~GB}$ \\
\hline & $\begin{array}{l}1 \mathrm{HR} \\
\text { Mud }\end{array}$ & & 269 & $\begin{array}{l}\text { Successf } \\
\mathrm{ul}\end{array}$ & 270 & & No & $2.30 \mathrm{hrs}$ & $\mathrm{F}$ & $1.0 \mathrm{~GB}$ \\
\hline & $\begin{array}{l}5 \mathrm{HR} \\
\text { mud }\end{array}$ & & 269 & $\begin{array}{l}\text { Successf } \\
\text { ul }\end{array}$ & 272 & & No & $1.58 \mathrm{hrs}$ & $\mathrm{F}$ & $1.0 \mathrm{~GB}$ \\
\hline & $\begin{array}{l}25 \mathrm{HR} \\
\text { mud }\end{array}$ & & 269 & $\begin{array}{l}\text { Successf } \\
\text { ul }\end{array}$ & 270 & & No & $2.10 \mathrm{hrs}$ & $\mathrm{F}$ & $1.0 \mathrm{~GB}$ \\
\hline
\end{tabular}

Method: Heating method

\begin{tabular}{|c|c|c|c|c|c|c|c|c|c|c|}
\hline \multirow[t]{4}{*}{ recuva } & $\begin{array}{l}\text {.No of } \\
\text { samples }\end{array}$ & $\begin{array}{l}\text { Types } \\
\text { of } \\
\text { files } \\
\text { in }\end{array}$ & $\begin{array}{l}\text { No files } \\
\text { before } \\
\text { recover }\end{array}$ & $\begin{array}{l}\text { Scannin } \\
\text { g status }\end{array}$ & $\begin{array}{l}\text { No } \\
\text { files } \\
\text { after } \\
\text { recov } \\
\text { er }\end{array}$ & $\begin{array}{l}\text { Noise } \\
\text { And type of noise\& } \\
\text { Reason of noise }\end{array}$ & $\begin{array}{l}\text { Data } \\
\text { loss }\end{array}$ & $\begin{array}{l}\text { Time } \\
\text { required for } \\
\text { deep } \\
\text { scanning }\end{array}$ & $\begin{array}{l}\text { Type of } \\
\text { recover } \\
\text { y } \\
\text { F/P/NO } \\
\text { recover } \\
\text { y }\end{array}$ & $\begin{array}{l}\text { Recovered } \\
\text { Data }\end{array}$ \\
\hline & $\begin{array}{l}\text { At } 80 \\
\text { degree } \\
\text { in hot } \\
\text { air oven }\end{array}$ & \multirow{3}{*}{$\begin{array}{l}\text { Webp, } \\
\text { dll,exe } \\
\text {,m4a, } \\
\text { mp3,j } \\
\text { pg,png } \\
\text {,pptx,d } \\
\text { ocs,pd } \\
\text { f,mkv, } \\
\text { mp4 }\end{array}$} & 269 & $\begin{array}{l}\text { Successf } \\
\text { ul }\end{array}$ & 288 & \multirow{2}{*}{$\begin{array}{l}\text { Types of noise: } \\
\text { Wed,To,TED,OY,O } \\
\text { UT,CESa,ITH,ION, } \\
\text { ING,ILE,IA,I<,HF } \\
\text { A,H<(E,H<,Fix,File } \\
\text {,EAD,ATE,ATA,A } \\
\text { ND,AFL,\%f",s",SD }\end{array}$} & No & $3.56 \mathrm{hr}$ & $\mathrm{F}$ & $29.5 \mathrm{~GB}$ \\
\hline & $\begin{array}{l}\text { At } 110 \\
\text { degree } \\
\text { in hot } \\
\text { air oven }\end{array}$ & & 269 & $\begin{array}{l}\text { Successf } \\
\text { ul }\end{array}$ & 285 & & No & $3.45 \mathrm{hrs}$ & $\mathrm{F}$ & $29.5 \mathrm{~GB}$ \\
\hline & $\begin{array}{l}\text { At } 160 \\
\text { degree } \\
\text { in hot } \\
\text { air oven }\end{array}$ & & 269 & \multicolumn{5}{|c|}{ Not Detected } & $\mathrm{N}$ & 00 \\
\hline
\end{tabular}




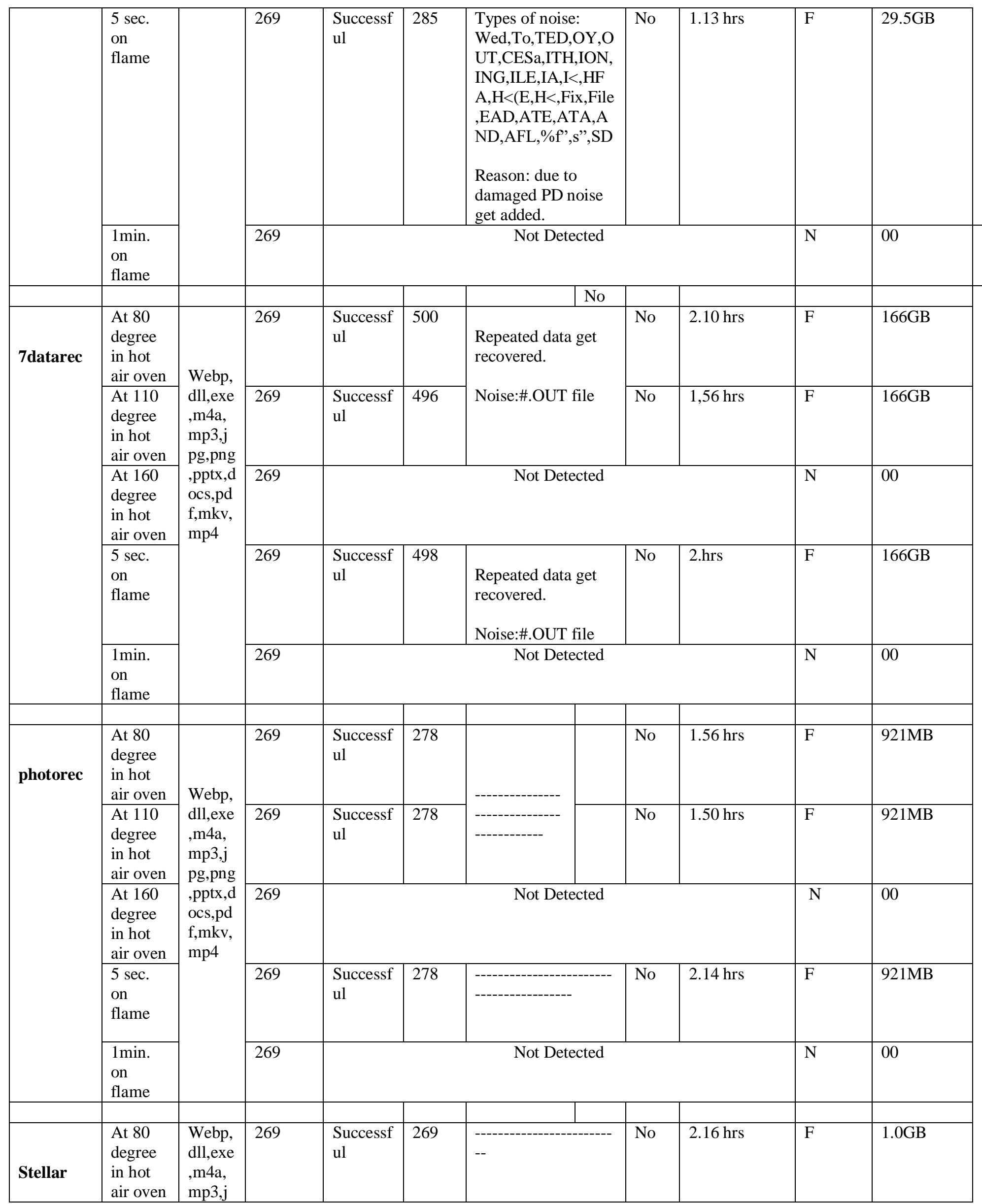


Determining the Probability of Recovering Data from Damaged USB Flash Drive

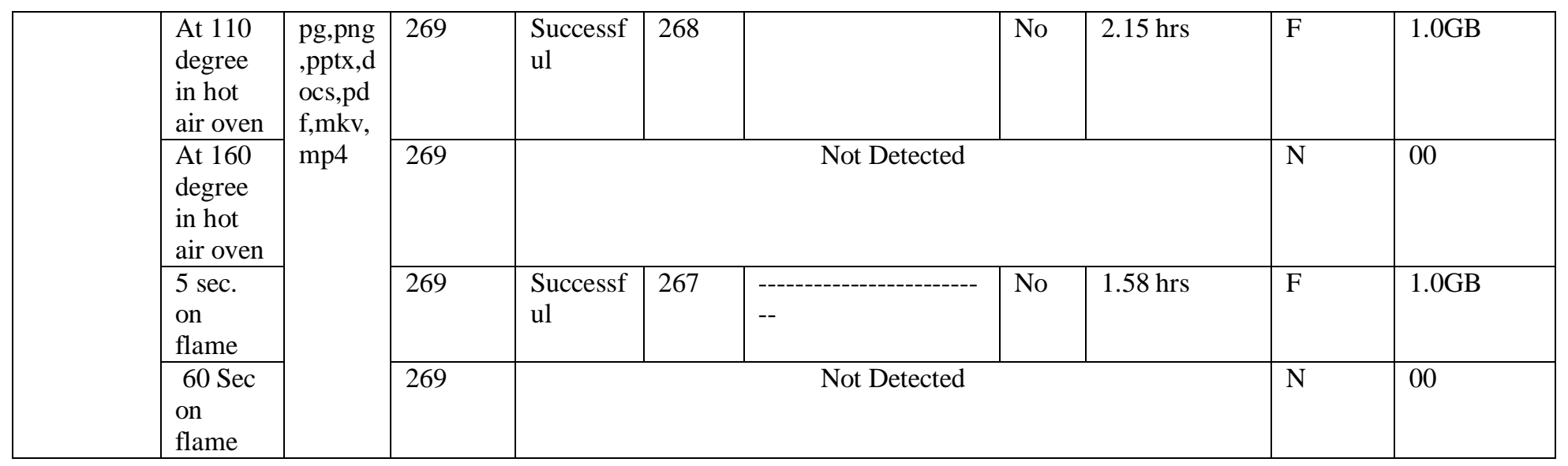

\section{Method: Freezing Method}

\begin{tabular}{|c|c|c|c|c|c|c|c|c|c|c|}
\hline \multirow{6}{*}{ recuva } & $\begin{array}{l}\text { No of } \\
\text { samples }\end{array}$ & $\begin{array}{l}\text { Types } \\
\text { of } \\
\text { files } \\
\text { in }\end{array}$ & $\begin{array}{l}\text { No of } \\
\text { files } \\
\text { before } \\
\text { recover }\end{array}$ & $\begin{array}{l}\text { Scannin } \\
\text { g status }\end{array}$ & $\begin{array}{l}\text { No files } \\
\text { after } \\
\text { recover }\end{array}$ & $\begin{array}{l}\text { Noise } \\
\text { And type of } \\
\text { noise \& } \\
\text { Reason of noise }\end{array}$ & $\begin{array}{l}\text { Data } \\
\text { loss }\end{array}$ & $\begin{array}{l}\text { Time } \\
\text { required } \\
\text { for deep } \\
\text { scanning }\end{array}$ & $\begin{array}{l}\text { Type of } \\
\text { recovery } \\
\text { F/P/NO } \\
\text { recovery }\end{array}$ & $\begin{array}{l}\text { Recovered } \\
\text { Data }\end{array}$ \\
\hline & $\begin{array}{l}1 \mathrm{hr} \\
\text { Freeze }\end{array}$ & \multirow{5}{*}{$\begin{array}{l}\text { Webp, } \\
\text { dll,exe } \\
\text {,m4a, } \\
\text { mp3,j } \\
\text { pg,png } \\
\text {,pptx,d } \\
\text { ocs,pd } \\
\text { f,mkv, } \\
\text { mp4 }\end{array}$} & 269 & $\begin{array}{l}\text { successf } \\
\text { ul }\end{array}$ & 288 & \multirow{5}{*}{$\begin{array}{l}\text { Types of noise: } \\
\text { Wed,To,TED,OY } \\
\text {,OUT,CESa,ITH,I } \\
\text { ON,ING,ILE,IA,I } \\
\text { <,HFA,H<(E,H<, } \\
\text { Fix,File,EAD,AT } \\
\text { E,ATA,AND,AF } \\
\text { L,\%f",s",SD }\end{array}$} & No & $1.56 \mathrm{hrs}$ & $\mathrm{F}$ & $29.5 \mathrm{~GB}$ \\
\hline & $\begin{array}{l}24 \mathrm{hr} \\
\text { freeze }\end{array}$ & & 269 & $\begin{array}{l}\text { Successf } \\
\text { ul }\end{array}$ & 286 & & Yes & $1.50 \mathrm{hrs}$ & $\mathrm{P}$ & $29.5 \mathrm{~GB}$ \\
\hline & $\begin{array}{l}48 \mathrm{hr} \\
\text { freeze }\end{array}$ & & 269 & $\begin{array}{l}\text { Successf } \\
\text { ul }\end{array}$ & 285 & & Yes & $1.57 \mathrm{hrs}$ & $\mathrm{P}$ & $29.5 \mathrm{~GB}$ \\
\hline & $\begin{array}{l}72 \mathrm{hr} \\
\text { freeze }\end{array}$ & & 269 & $\begin{array}{l}\text { Successf } \\
\text { ul }\end{array}$ & 285 & & Yes & $2.14 \mathrm{hrs}$ & $\mathrm{P}$ & $29.5 \mathrm{~GB}$ \\
\hline & $\begin{array}{l}96 \mathrm{hr} \\
\text { freeze }\end{array}$ & & 269 & $\begin{array}{l}\text { Successf } \\
\text { ul }\end{array}$ & 267 & & Yes & $1.45 \mathrm{hrs}$ & $\mathrm{P}$ & $6.62 \mathrm{~GB}$ \\
\hline \multirow{5}{*}{ 7datarec } & $\begin{array}{l}1 \mathrm{HR} \\
\text { Freeze }\end{array}$ & \multirow{5}{*}{$\begin{array}{l}\text { Webp, } \\
\text { dll,exe } \\
\text {,m4a, } \\
\text { mp3,j } \\
\text { pg,png } \\
\text {,pptx,d } \\
\text { ocs,pd } \\
\text { f,mkv, } \\
\text { mp4 } \\
\end{array}$} & 269 & $\begin{array}{l}\text { Successf } \\
\text { ul }\end{array}$ & 501 & \multirow{5}{*}{$\begin{array}{l}\text { Repeated data get } \\
\text { recovered. } \\
\text { Noise:\#.OUT file }\end{array}$} & No & $2.15 \mathrm{hrs}$ & $\mathrm{F}$ & $1.67 \mathrm{~GB}$ \\
\hline & $\begin{array}{l}24 \mathrm{HR} \\
\text { freeze }\end{array}$ & & 269 & $\begin{array}{l}\text { Successf } \\
\text { ul }\end{array}$ & 499 & & No & $1.56 \mathrm{hrs}$ & $\mathrm{F}$ & $1.67 \mathrm{~GB}$ \\
\hline & $\begin{array}{l}48 \mathrm{hr} \\
\text { freeze }\end{array}$ & & 269 & $\begin{array}{l}\text { Successf } \\
\text { ul }\end{array}$ & 498 & & No & $1.59 \mathrm{hrs}$ & $\mathrm{F}$ & $1.67 \mathrm{~GB}$ \\
\hline & $\begin{array}{l}72 \mathrm{hr} \\
\text { freeze }\end{array}$ & & 269 & $\begin{array}{l}\text { Successf } \\
\text { ul }\end{array}$ & 498 & & No & $2.19 \mathrm{hrs}$ & $\mathrm{F}$ & $1.67 \mathrm{~GB}$ \\
\hline & $\begin{array}{l}96 \mathrm{hr} \\
\text { freeze }\end{array}$ & & 269 & $\begin{array}{l}\text { Successf } \\
\text { ul }\end{array}$ & 505 & & No & $2.10 \mathrm{hrs}$ & $\mathrm{F}$ & $1.67 \mathrm{~GB}$ \\
\hline \multirow{6}{*}{ photorec } & $\begin{array}{l}1 \mathrm{HR} \\
\text { Freeze }\end{array}$ & \multirow{5}{*}{$\begin{array}{l}\text { Webp, } \\
\text { dll,exe } \\
\text {,m4a, } \\
\text { mp3,j } \\
\text { pg,png } \\
\text {,pptx,d } \\
\text { ocs,pd } \\
\text { f,mkv, } \\
\text { mp4 }\end{array}$} & 269 & $\begin{array}{l}\text { Successf } \\
\text { ul }\end{array}$ & 278 & \multirow{5}{*}{-------------------------- } & No & $1.56 \mathrm{hrs}$ & $\mathrm{F}$ & $921 \mathrm{MB}$ \\
\hline & $\begin{array}{l}24 \mathrm{HR} \\
\text { freeze }\end{array}$ & & 269 & $\begin{array}{l}\text { Successf } \\
\text { ul }\end{array}$ & 277 & & No & $1.50 \mathrm{hrs}$ & $\mathrm{F}$ & $921 \mathrm{MB}$ \\
\hline & $\begin{array}{l}48 \mathrm{hr} \\
\text { freeze }\end{array}$ & & 269 & $\begin{array}{l}\text { Successf } \\
\text { ul }\end{array}$ & 277 & & No & $1.57 \mathrm{hrs}$ & $\mathrm{F}$ & $921 \mathrm{MB}$ \\
\hline & $\begin{array}{l}72 \mathrm{hr} \\
\text { freeze }\end{array}$ & & 269 & $\begin{array}{l}\text { Successf } \\
\text { ul }\end{array}$ & 277 & & No & $2.14 \mathrm{hrs}$ & $\mathrm{F}$ & $921 \mathrm{MB}$ \\
\hline & $\begin{array}{l}96 \mathrm{hr} \\
\text { freeze }\end{array}$ & & 269 & $\begin{array}{l}\text { Successf } \\
\text { ul }\end{array}$ & 269 & & Yes & $1.45 \mathrm{hrs}$ & $\mathrm{P}$ & $921 \mathrm{MB}$ \\
\hline & & & & & & & & & & \\
\hline
\end{tabular}




\begin{tabular}{|c|c|c|c|c|c|c|c|c|c|c|}
\hline \multirow{5}{*}{ Stellar } & $\begin{array}{l}1 \mathrm{HR} \\
\text { Freeze }\end{array}$ & \multirow{5}{*}{$\begin{array}{l}\text { Webp, } \\
\text { dll,exe } \\
\text {,m4a, } \\
\text { mp3,j } \\
\text { pg,png } \\
\text {,pptx,d } \\
\text { ocs,pd } \\
\text { f,mkv, } \\
\text { mp4 }\end{array}$} & 269 & $\begin{array}{l}\text { Successf } \\
\text { ul }\end{array}$ & 264 & \multirow[t]{5}{*}{\begin{tabular}{|l}
----------------- \\
---
\end{tabular}} & Yes & $2.16 \mathrm{hrs}$ & $\mathrm{F}$ & $996 \mathrm{MB}$ \\
\hline & $\begin{array}{l}24 \mathrm{HR} \\
\text { freeze }\end{array}$ & & 269 & $\begin{array}{l}\text { Successf } \\
\text { ul }\end{array}$ & 262 & & Yes & $2.15 \mathrm{hrs}$ & $\mathrm{F}$ & 997MB \\
\hline & $\begin{array}{l}48 \mathrm{hr} \\
\text { freeze }\end{array}$ & & 269 & $\begin{array}{l}\text { Successf } \\
\text { ul }\end{array}$ & 263 & & Yes & $2.30 \mathrm{hrs}$ & $\mathrm{F}$ & $0.97 \mathrm{~GB}$ \\
\hline & $\begin{array}{l}72 \mathrm{hr} \\
\text { freeze }\end{array}$ & & 269 & $\begin{array}{l}\text { Successf } \\
\text { ul }\end{array}$ & 263 & & Yes & $1.58 \mathrm{hrs}$ & $\mathrm{F}$ & 999MB \\
\hline & $\begin{array}{l}96 \mathrm{hr} \\
\text { freeze }\end{array}$ & & 269 & $\begin{array}{l}\text { Successf } \\
\text { ul }\end{array}$ & 265 & & Yes & $2.10 \mathrm{hrs}$ & $\mathrm{F}$ & $0.99 \mathrm{~GB}$ \\
\hline
\end{tabular}

Method: Scratch Method

\begin{tabular}{|c|c|c|c|c|c|c|c|c|c|c|}
\hline \multirow[t]{6}{*}{ recuva } & \begin{tabular}{|l|} 
No of \\
samples
\end{tabular} & $\begin{array}{l}\text { Types } \\
\text { of } \\
\text { files } \\
\text { in }\end{array}$ & $\begin{array}{l}\text { No files } \\
\text { before } \\
\text { recover }\end{array}$ & $\begin{array}{l}\text { Scannin } \\
\text { g status }\end{array}$ & $\begin{array}{l}\text { No files } \\
\text { after } \\
\text { recover }\end{array}$ & $\begin{array}{l}\text { Noise } \\
\text { And type of } \\
\text { noise \& } \\
\text { Reason of noise }\end{array}$ & $\begin{array}{l}\text { Data } \\
\text { loss }\end{array}$ & $\begin{array}{l}\text { Time } \\
\text { required for } \\
\text { deep } \\
\text { scanning }\end{array}$ & $\begin{array}{l}\text { Type of } \\
\text { recover } \\
\text { y } \\
\text { F/P/NO } \\
\text { recover } \\
\text { y }\end{array}$ & $\begin{array}{l}\text { Recovered } \\
\text { Data }\end{array}$ \\
\hline & 100 & \multirow{5}{*}{$\begin{array}{l}\text { Webp, } \\
\text { dll,exe } \\
\text {,m4a, } \\
\text { mp3,j } \\
\text { pg,png } \\
\text {,pptx,d } \\
\text { ocs,pd } \\
\text { f,mkv, } \\
\text { mp4 }\end{array}$} & 269 & $\begin{array}{l}\text { successf } \\
\text { ul }\end{array}$ & 271 & \multirow{3}{*}{$\begin{array}{l}\text { Types of noise: } \\
\text { Wed,To,TED,OY } \\
\text {,OUT,CESa,ITH,I } \\
\text { ON,ING,ILE,IA,I } \\
\text { <,HFA,H<(E,H<, } \\
\text { Fix,File,EAD,AT } \\
\text { E,ATA,AND,AF } \\
\text { L,\%f',s",SD } \\
\text { Reason: due to } \\
\text { damaged PD } \\
\text { noise get added. }\end{array}$} & Yes & $1.56 \mathrm{hrs}$ & $\mathrm{P}$ & $6.63 \mathrm{~GB}$ \\
\hline & $\begin{array}{ll}150 \\
\end{array}$ & & 269 & $\begin{array}{l}\text { successf } \\
\text { ul }\end{array}$ & 287 & & No & $1.50 \mathrm{hrs}$ & $\mathrm{F}$ & $29.5 \mathrm{~GB}$ \\
\hline & 200 & & 269 & $\begin{array}{l}\text { Successf } \\
\text { ul }\end{array}$ & 268 & & Yes & $1.57 \mathrm{hrs}$ & $\mathrm{P}$ & $6.62 \mathrm{~GB}$ \\
\hline & 250 & & 269 & \multicolumn{5}{|c|}{ Not Detected } & $\mathrm{N}$ & 00 \\
\hline & 500 & & 269 & \multicolumn{5}{|c|}{ Not Detected } & $\mathrm{N}$ & 00 \\
\hline \multirow{5}{*}{ 7datarec } & 100 & \multirow{5}{*}{$\begin{array}{l}\text { Webp, } \\
\text { dll,exe } \\
\text {,m4a, } \\
\text { mp3,j } \\
\text { pg,png } \\
\text {,pptx,d } \\
\text { ocs,pd } \\
\text { f,mkv, } \\
\text { mp4 }\end{array}$} & 269 & $\begin{array}{l}\text { successf } \\
\text { ul }\end{array}$ & 498 & \multirow{3}{*}{$\begin{array}{l}\text { Repeated data get } \\
\text { recovered. } \\
\text { Noise:\#.OUT file }\end{array}$} & No & $2.15 \mathrm{hrs}$ & $\mathrm{F}$ & $1.66 \mathrm{~GB}$ \\
\hline & 150 & & 269 & $\begin{array}{l}\text { successf } \\
\text { ul }\end{array}$ & 496 & & No & $1.56 \mathrm{hrs}$ & $\mathrm{F}$ & $1.65 \mathrm{~GB}$ \\
\hline & 200 & & 269 & $\begin{array}{l}\text { Successf } \\
\text { ul }\end{array}$ & 497 & & No & $1.59 \mathrm{hrs}$ & $\mathrm{F}$ & $1.65 \mathrm{~GB}$ \\
\hline & 250 & & 269 & \multicolumn{5}{|c|}{ Not Detected } & $\mathrm{N}$ & 00 \\
\hline & 500 & & 269 & \multicolumn{5}{|c|}{ Not Detected } & $\mathrm{N}$ & 00 \\
\hline \multirow{5}{*}{ Photorec } & 100 & \multirow{5}{*}{$\begin{array}{l}\text { Webp, } \\
\text { dll,exe } \\
\text {,m4a, } \\
\text { mp3,j } \\
\text { pg,png } \\
\text {,pptx,d } \\
\text { ocs,pd } \\
\text { f,mkv, } \\
\text { mp4 }\end{array}$} & 269 & $\begin{array}{l}\text { successf } \\
\mathrm{ul}\end{array}$ & 279 & & No & $1.56 \mathrm{hrs}$ & $\mathrm{F}$ & $921 \mathrm{MB}$ \\
\hline & 150 & & 269 & $\begin{array}{l}\text { successf } \\
\text { ul }\end{array}$ & 277 & & No & $1.56 \mathrm{hrs}$ & $\mathrm{F}$ & $921 \mathrm{MB}$ \\
\hline & 200 & & 269 & $\begin{array}{l}\text { Successf } \\
\text { ul }\end{array}$ & 277 & & No & $1.50 \mathrm{hrs}$ & $\mathrm{F}$ & $921 \mathrm{MB}$ \\
\hline & 250 & & 269 & \multicolumn{5}{|c|}{ Not Detected } & $\mathrm{N}$ & 00 \\
\hline & 500 & & 269 & \multicolumn{5}{|c|}{ Not Detected } & $\mathrm{N}$ & 00 \\
\hline
\end{tabular}


Determining the Probability of Recovering Data from Damaged USB Flash Drive

\begin{tabular}{|c|c|c|c|c|c|c|c|c|c|c|}
\hline \multirow{5}{*}{ Stellar } & 100 & \multirow{5}{*}{$\begin{array}{l}\text { Webp, } \\
\text { dll,exe } \\
\text {,m4a, } \\
\text { mp3,j } \\
\text { pg,png } \\
\text {,pptx,d } \\
\text { ocs,pd } \\
\text { f,mkv, } \\
\text { mp4 }\end{array}$} & 269 & $\begin{array}{l}\text { successf } \\
\text { ul }\end{array}$ & 267 & \multirow[t]{3}{*}{ 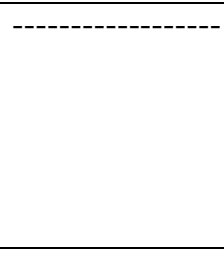 } & $\mathrm{NO}$ & $1.45 \mathrm{hrs}$ & $\mathrm{F}$ & $1.0 \mathrm{~GB}$ \\
\hline & 150 & & 269 & $\begin{array}{l}\text { successf } \\
\mathrm{ul}\end{array}$ & 265 & & NO & $2.15 \mathrm{hrs}$ & $\mathrm{F}$ & 0.99GB \\
\hline & 200 & & 269 & $\begin{array}{l}\text { successf } \\
\mathrm{ul}\end{array}$ & 267 & & $\mathrm{NO}$ & $2.30 \mathrm{hrs}$ & $\mathrm{F}$ & $0.99 \mathrm{~GB}$ \\
\hline & 250 & & 269 & \multicolumn{5}{|c|}{ Not Detected } & $\mathrm{N}$ & 00 \\
\hline & 500 & & 269 & \multicolumn{5}{|c|}{ Not Detected } & $\mathrm{N}$ & 00 \\
\hline
\end{tabular}

\section{RESULT AND CONCLUSION:}

Physical damaged was done by applying Various Methods like Buried in soil, Deeping in aqueous media like water, Heating, Freezing Scratching to the PD with various criteria and conditions and then during recovery of data is recovered in successful recovery, Partially Sucessful Recovery and unsuccessful Recovery. Unsuccessful recovery was happened at very extreme conditions when applied.It is concluded that stellar phoenix as it was licensed software gives 100\% result with little noise insertion. While other three software are observed maximum noise is inserted because of which file properties like file size changed, memory space changed. One disadvantage was that more memory size to store so many amounts of data is required

\section{REFERENCES:-}

1. Recuva software https://recuva.en.softonic.com/downloadStellar Phoneix

2. 7 Data Recovery Software https://7datarecovery.com/\#forwardPhotrec data recovery

3. Photorec $7.0 \quad$ Data $\quad$ Recovery https://downloads.tomsguide.com/PhotoRec,0301-32874.html

4. Stellar Phoneix : https://www.stellarinfo.com/

5. https://www.google.com/search?rlz=1C1CHBD_enIN764IN764\& biw $=1200 \& b i h=733 \&$ tbm $=i s c h \& s a=1 \&$ ei $=$ s9yIXMGYD9q7rQH TtrnABw\&q=images+of+smart+toy+pen+drive+\&oq=images+of + smart+toy+pen+drive+\&gs_l=img.3...9508.9508..10000...0.0..0. 148.148.0j1 ...........1..gws-wiz-img.IOMhle9HT8\#imgrc=4e9pT38M_LYKGM:[

6. https://www.google.com/search?rlz=1C1CHBD_enIN764IN764\& biw $=1200 \&$ bih $=733 \&$ tbm $=$ isch \&sa $=1 \&$ ei $=$ Z9eIXKuIGZa9rQHi w7eABA\&q=images+of+smart+toy+pen+drive++bracelets\&oq=i mages+of+smart+toy+pen+drive++bracelets\&gs_l=img.3...11277 .16608..16992...0.0..0.1263.2965.0j9j0j1j7-1..........1..gws-wizimg.RNbWXgRt-eI\#imgrc=vW9w3N0V6az4CM:

7. https://www.google.com/search?rlz=1C1CHBD enIN764IN764\& biw=1200\&bih=733\&tbm=isch\&sa=1\&ei=Z9eIXKuIGZa9rQHi w7eABA\&q=images+of+smart+toy+pen+drive+\&oq=images+of + smart+toy+pen+drive+\&gs_l=img.3 ...4164.4164..4496...0.0..0.0 .0..........1..gws-wizimg.GpD7xMICc2E\#imgrc $=$ uGg 4WVwMi R1M:

8. https://www.google.com/search?rlz=1C1CHBD_enIN764IN764\& biw=1200\&bih=733\&tbm=isch\&sa=1\&ei=Z9eIXKuIGZa9rQHi w7eABA\&q=images+of+smart+toy+pen+drive++bracelets\&oq $=\mathrm{i}$ mages+of+smart+toy+pen+drive++bracelets\&gs_l=img.3 ...11277 16608..16992...0.0..0.1263.2965.0j9j0j1j7-1.....0....1..gws-wizimg.RNbWXgRt-eI\#imgrc=vW9w3N0V6az4CM:

9. https://www.google.com/search?rlz=1C1CHBD_enIN764IN764\& biw=1200\&bih=733\&tbm=isch\&sa=1\&ei=Z9eIXKuIGZa9rQHi w7eABA\&q=images+of+smart+toy+pen+drive+\&oq=images+of

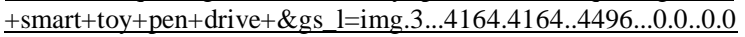
.0...........1..gws-wizimg.GpD7xMICc2E\#imgrc=_2MGQbESmY_QvM:

10. https://www.wantitall.co.za/pchardware/8gb-usb2-0-memorystick-creative-metal-wrench-usb-flash-drive-kepmem-funny-giftpen-drive b06xv88cj7

11. Sneha Pandhare, Dr.Shobha Bawiskar," Recovery Of Data From Damaged Disks".(Online-Oral Presentation), International Conference on "Innovations in Engineering, Technology and Sciences"- (ICIETS2018) with catlog "CFP18Q63-PRJ:978-15386-7321-8" held on September 21-22,2018, NIE Institute of Technology, Mysore, Karnataka, (Bangalore)India, will be published in IEEE Xplore Digital Library

12. Alpna, Dr. Sona Malhotra " Cyber Crime-Its Types, Analysis and Prevention Techniques" International Journal of Advanced Research in Computer Science and Software Engineering
Research Volume 6, Issue 5, May 2016 ISSN: 2277 128X ,page no 145. Paper Available online at: www.ijarcsse.com

13. Madison, Alex (2016-07-09). "Keychain Not Included: The Five Highest-Capacity USB Flash Drives for Your Digital Life". Digital Trends. Retrieved 17 October 2016.

14. Jump up to:a b Athow, Desire (2016-07-04). "The best USB flash drives 2016". Tech Radar. Retrieved 17 October 2016.

15. "The Largest Flash Drives | Digital Trends". Digital Trends. 201807-23. Retrieved 2018-10-09.

16. G. I. A. Incorporated, "USB Flash Drive Market Trends," Global Industry Analyst Inc., $20 \quad$ March 2017.[Online].Available:http://www.strategyr.com/MarketRe search/USB_Flash_Drives_Market_Trends.asp. [Accessed 20 March 2017

17. Parthasarathy, M., \& Parthasarathy, S. (2017). Performance Analysis of USB Flash Memory Devices on Linux vs. Windows XP.

18. JUANCHO D. ESPINELI, 2 JASMIN NIQUIDULA, "INFORMATION THEORY IN USB FLASH MEMORY DEVICE ANALYSIS" Proceedings of Academics World $63 \mathrm{rd}$ International Conference, Manila, Philippines, 28th -29th April 2017, http://www.worldresearchlibrary.org/up_proc/pdf/83414997520531-6.pdf

19. International Journal of Engineering Research and Development. ISSN: 2278-067X. 2012; 1(6): 25-34.

20. 20 B Naresh Kumar Reddy, N Venktram, Sireesha, "An Efficient Data Transmission by using Modern USB Flash Drive" International Journal of Electrical and Computer Engineering (IJECE) Vol. 4, No. 5, October 2014, pp. 730 740 ISSN: 2088 8708

21. Oka Mahendra,Djohar Syamsi,Ade Ramdan,Marcella Astrid,"Design and implementation of data storage system using USB flash drive in a microcontroller based data logger" , DOI 10.1109/ICACOMIT.2015.7440175,Electronic ISBN: 978-14673-7408-8 CD-ROM ISBN: 978-1-4673-74071,https://ieeexplore.ieee.org/abstract/document/7440175.

22. PNY USB Flash Drive - CES 2006 - LetsGoDigital. Cesshow.com. Retrieved on 2011-05-18.

23. BlueTrek Bizz - an expandable USB and a Bluetooth headset in one Archived 2014-08-29 at the Wayback Machine. TechChee.com (2008-05-20). Retrieved on 2011-05-18. 


\section{AUTHORS PROFILE}

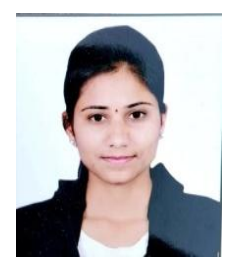

I am kalpana Dnyaneshwar Shinde Student of M.Sc (Forensic Science)-SY-IV ${ }^{\text {th }}$ semester in Government Institute of Forensics Science Aurangabad in Department of Digital and Cyber Forensics. I had done my research work determine the possibility of recovering the data from damaged USB Flash Drive. Other topic of are cyber investigation, incidence response, network Forensic, Multimedia Forensics, etc... further wish to apply for Ph.D too.

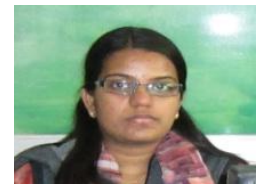

Kale Vini Arun Completed M.Sc (Computer Science) in 2008 from University of Pune. Qualified UGC-NET in "Computer Science and Applications" held on 24th June 2012. work experience is of six plus years - includes as Lecturer in K.V.N.Naik's Arts, Commerce and Science College, as Junior Consultant Yashwantrao Chavan Maharashtra Open University, Nashik, as Assistant Professor in MVP's KSKW College, Nasik and Currently working as Assistant Professor in Government Institute of Forensic Science. Aurangabad. Topic of research interest includes Digital and Cyber forensic, Mobile Forensics, Network forensics, Nanotechnology, image processing, Internet Of Things

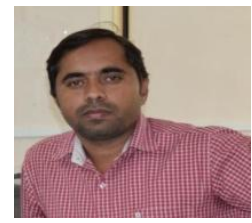

Dr.Charansing Nathusing Kayte Bachelor of Science in PCM (BSc) from Yashwantrao Chavan College of Science Sillod, Aurangabad, affiliated to Dr.Babasaheb Ambedkar Marathwada University, Aurangabad Maharashtra, Master of Science in Information Technology (MSc) Dr.Babasaheb Ambedkar Marathwada University, Aurangabad, Maharashtra, India., Ph.D in Computer Science Singhania University,Rajasthan,India. 13 plus years of teaching experience, have published 30 plus research articles in various national / international conferences, Journals, Seminars. Currently working as HOD of Digital and Cyber forensic in Government Institute of Forensic Science. Aurangabad. Topic of research interest includes Digital and Cyber forensic, Mobile Forensics, Network forensics, image processing, and Internet of Things Speech recognition.

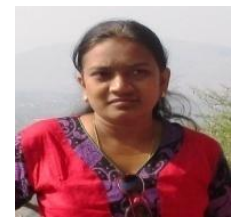

Dr.Shobha Bawiskar has completed BCS degree and M.Sc Computer Science from College of IT \& M, Vivekanand college of Arts, science and commerce respectively affiliated to Dr.Babasaheb Marathwada University, M.Phil from YCMOU from affiliated IMTR study centre in Aurangabad and received Ph.D in Computer Science from Dr.Babasaheb Marathwada University. 13 years of teaching experience. Have published twenty five plus research articles in various national / international conferences, Journals, Seminars. Currently working as Assistance Professor in Government Institute of Forensic Science. Aurangabad. Topic of research interest includes Digital and Cyber forensic, Mobile Forensics, Network forensics, Nanotechnology, image processing,Internet Of Things. 